\title{
Hand to Mouth: Automatic Imitation Across Effector Systems
}

\author{
Jane Leighton \\ University College London
}

\author{
Cecilia Heyes \\ University of Oxford
}

\begin{abstract}
The effector dependence of automatic imitation was investigated using a stimulus-response compatibility (SRC) procedure during which participants were required to make an open or closed response with their hand or their mouth. The correct response for each trial was indicated by a pair of letters in Experiments 1 and 2 and by a colored square in Experiment 3. Each of these imperative stimuli was accompanied by task-irrelevant action images depicting a hand or mouth opening or closing. In relation to the response, the irrelevant stimulus was movement compatible or movement incompatible, and effector compatible or effector incompatible. A movement compatibility effect was observed for both hand and mouth responses. These movement compatibility effects were present when the irrelevant stimulus was effector compatible and when it was effector incompatible, but were smaller when the irrelevant stimulus and response effectors were incompatible. Consistent with the associative sequence learning (ASL) model of imitation, these findings indicate that automatic imitation is partially effector dependent and therefore that the effector dependence of intentional imitation reflects, at least in part, the nature of the mechanisms that mediate visuomotor translation for imitation.
\end{abstract}

Keywords: automatic imitation, effector, associative sequence learning (ASL), mirror neuron

Instructed imitation is usually effector dependent. In everyday life and in the laboratory, when a person is asked to imitate an action, he or she reproduces the trajectory or topography of the modeled movement by using the same part of the body (Bird, Brindley, Leighton, \& Heyes, 2007; Franz, Ford, \& Werner, 2007; Head, 1920; Heyes \& Ray, 2004; Wapner \& Cirillo, 1968; Wohlschläger, Gattis \& Bekkering, 2003). Hand movements are imitated with the hands, foot movements with the feet, and mouth movements with the mouth. The purpose of the present study was to find out whether the effector dependence of instructed imitation reflects the structure of the core neurocognitive mechanisms of imitation and, if so, what it can tell us about those mechanisms.

The core mechanisms of imitation are those that solve the “correspondence problem" (Brass \& Heyes 2005), translating visual input from observed body movements into matching motor output. It is possible that these mechanisms are effector dependent; for example, they may be incapable of translating observed movements of one effector system into motor output in another effector system. If so, then effector dependence at the mechanism level could be responsible for the effector dependence observed in

This article was published Online First August 23, 2010.

Jane Leighton, Department of Psychology, University College London; and Cecilia Heyes, All Souls College and Department of Experimental Psychology, University of Oxford.

This research was supported by a Medical Research Council (MRC) doctoral studentship awarded to Jane Leighton, the Economic and Social Research Council (ESRC) research centre for Economic Learning and Social Evolution (ELSE), and the European Community's Sixth Framework Programme under contract number NEST 012929.

Correspondence concerning this article should be addressed to Cecilia Heyes, All Souls College and Department of Experimental Psychology, University of Oxford, Oxford OX1 4AL, United Kingdom. E-mail: cecilia.heyes@all-souls.ox.ac.uk instructed imitation performance. Alternatively, it might be that the core mechanisms of imitation are fully or partially effector independent, and that their output is filtered by cultural knowledge. This hypothesis suggests that, within anatomical constraints, a participant observing a hand movement is enabled by core imitation mechanisms to reproduce the movement with his or her hand or with an alternative effector system. However, if the participant has been told to "do this" or "imitate," she chooses to use the same effector as the model because she understands these instructions, within the conventions of her language and social group, to require both movement and effector matching.

Studies of imitation in infancy suggest that the effector dependence of instructed imitation is not purely conventional. In infants too young to be given instructions, observation of tongue protrusion increases the probability of tongue protrusion, but not of lip protrusion (Meltzoff \& Moore, 1977, 1983, 1989, 1997). Given the age of the infants in these studies (some as young as $72 \mathrm{hr}$ ), it is highly improbable that their behavior was guided by inferences about what was required, or what would be expedient, in the test situation. However, these findings do not provide conclusive evidence that the mechanisms of imitation are effector dependent, because the tendency of infants to respond to tongue protrusion with tongue protrusion may be mediated by arousal processes, rather than by the mechanisms that mediate imitation later in development (Anisfeld, 1991, 1996; Jones, 1996, 2006; Ullstadius, 1998).

As with infancy research, some recent studies of "automatic imitation" in adults provide suggestive, but not conclusive, evidence that the mechanisms of imitation are effector dependent. Performance in automatic imitation tasks is unlikely to be influenced by inferences about the experimenter's expectations or expediency because they examine imitation under conditions in which participants are not told to imitate, may not be aware that they are imitating, and in which imitation can interfere with the 
execution of task instructions (e.g. Kerzel \& Bekkering, 2000; Brass, Bekkering, Wohlschläger, \& Prinz, 2000; Craighero, Fadiga, Rizzolatti, \& Umilta, 1998; Heyes, Bird, Johnson, \& Haggard, 2005; Lakin \& Chartrand, 2003; Stanley, Gowen, \& Miall, in press; Stürmer, Aschersleben, \& Prinz, 2000). In a study of this kind, Chartrand and Bargh (1999) found that, when a model and an observer were in conversation, observation of face rubbing elicited more face rubbing than foot shaking, and vice versa for observation of foot shaking. Similarly, in a choice reaction time (RT) task, Bach and Tipper (2007) asked participants to identify a model as either George or John by pressing a button with their foot or with their hand, and found that observation of the model performing foot actions (kicking a ball) facilitated foot responses, whereas observation of the model performing hand actions (typing on a keyboard) facilitated hand responding. These studies, and others like them (Berger \& Hadley, 1975; Bertenthal, Longo, \& Kosobud, 2006; Brass et al., 2000; Gillmeister, Catmur, Brass, \& Heyes, 2010), suggest that observation of an effector in motion selectively activates responses made with the same effector. However, they do not tell us whether this "effector priming" effect is specific to movements that match those observed. Therefore, although effector priming is of interest in its own right, its occurrence does not necessarily imply that the mechanisms mediating movement imitation are effector dependent. To find out whether this is the case, it would be necessary to dissociate movement type (e.g., rubbing vs. shaking, kicking vs. typing) from effector type (e.g., feet vs. hands), and to show that observation of a particular movement type is more likely to elicit an imitative response when the response is performed with the modeled effector than when it is performed with an alternative effector. This logic was applied in the present study.

We investigated the effector dependence of imitation using a stimulus-response compatibility (SRC) automatic imitation procedure (Bekkering \& Kerzel, 2000; Bertenthal et al., 2006; Brass et al., 2000; Heyes et al., 2005; Kilner, Paulignan, \& Blakemore, 2003; Press, Bird, Flach, \& Heyes, 2005; Stürmer et al., 2000). In each trial of the choice RT task, participants were required to make one of four responses: to open their hand, to close their hand, to open their mouth, or to close their mouth. The correct response for each trial was indicated by a pair of letters (Experiments 1 and 2) or a colored square (Experiment 3) presented on a computer screen. The imperative stimuli were accompanied by one of four, task-irrelevant action stimuli: photographic images of an open hand, a closed hand, an open mouth, or a closed mouth. Thus, the irrelevant action stimulus and the correct response were either effector compatible and movement compatible (e.g., open-hand stimulus and open-hand response), effector compatible and movement incompatible (e.g., closed-hand stimulus and open-hand response), effector incompatible and movement compatible (e.g., open-mouth stimulus and open-hand response), or effector incompatible and movement incompatible (e.g., closed-mouth stimulus and an open-hand response).

Previous SRC studies, in which all responses were effector compatible, have shown a movement compatibility effect for hand opening and closing responses; for example, hand opening is initiated faster in response to a hand-opening stimulus than to a hand-closing stimulus (Heyes et al., 2005; Stürmer et al., 2000). They have also shown that this effect is not due to left-right or orthogonal spatial relations between the stimuli and responses
(Press, Bird, Walsh, \& Heyes, 2008). Therefore, in the present study, we expected to find a movement compatibility effect when the stimulus and the response effector were compatible. The primary focus of interest was the relative magnitude of any movement compatibility effect in the effector-compatible and the effector-incompatible conditions. If imitation mechanisms are effector independent, then the movement compatibility effect should be equally strong in the effector-compatible and effectorincompatible conditions. This would be a surprising result, given previous evidence of effector priming, but it would be interpretable: It would suggest that the effector dependence of instructed imitation is conventional or, more broadly, a product of high-level cognitive processes filtering the output from core mechanisms of imitation. ${ }^{1}$ If imitation mechanisms are partially effector dependent, one would expect the movement compatibility effect to be present in both the effector-compatible and the effectorincompatible conditions, but to be smaller in the latter condition. Finally, if these mechanisms are wholly effector dependent, then the movement compatibility effect should be present in the effector-compatible condition but absent in the effectorincompatible condition.

\section{Experiment 1}

Participants were instructed to make one of four responses to letters on a computer screen. They were told to open their mouth if the letters were $\mathrm{om}$, to close their mouth in response to $\mathrm{cm}$, to open their hand in response to $o h$, and to close their hand in response to $\mathrm{ch}$. Participants were also told that the letters would appear with irrelevant images of hand and mouth movements, which they should ignore. The irrelevant stimuli depicted a hand either opening or closing or a mouth opening or closing. Therefore, for any given trial, the response effector was either compatible or incompatible with the irrelevant stimulus effector and the response movement was either compatible or incompatible with the irrelevant stimulus movement. There were therefore two types of compatibility (effector and movement), and each had two levels (compatible and incompatible).

\section{Method}

Participants. Thirteen consenting, healthy participants with an average age of 25.7 years, 7 men, were recruited from the University College London Department of Psychology database and paid a small honorarium for their participation. All were right-handed, had normal or corrected-to-normal vision, and were proficient in the English language. They were naïve with respect to the purpose of

\footnotetext{
${ }^{1}$ Even if the present study found evidence of complete effector independence (e.g., that a mouth-opening stimulus facilitates a hand-opening response as much as it facilitates a mouth-opening response), the movement compatibility effect would still constitute an example of imitation rather than of emulation (Tomasello, 1996). In emulation, behavior is influenced, not by body movement stimuli, but by observation of the movement of an object of transitive action. In contrast, because we examined intransitive actions and controlled for spatial compatibility, any movement compatibility effect in the present study would indicate imitation; that observation of body movement stimuli facilitates performance of similar body movements.
} 
the experiment. The experiment was performed with local ethical committee approval and in accordance with the ethical standards laid down in the 1964 Declaration of Helsinki.

Stimuli and apparatus. All stimuli were presented on an LCD laptop computer screen $(60 \mathrm{~Hz}, 400 \mathrm{~mm}, 96 \mathrm{DPI})$ in color on a black background. Viewing was unrestrained at a distance of approximately $600 \mathrm{~mm}$. Warning stimuli consisted of photographic images of two effectors side by side, a mouth and a right hand, each in a neutral posture (see Figure 1a). In half of the trials the hand was on the left of the screen with the mouth on the right, and in the other half, this configuration was reversed.

The imperative stimulus consisted of two letters in the center of the screen, which indicated which movement to make. These letters were accompanied by task-irrelevant stimuli, consisting of the hand and mouth images in the same left-right position as in the preceding warning stimulus, but one effector was displayed in an open or closed posture, whereas the other remained in the neutral posture. Presentation of the warning stimulus was immediately followed by the imperative and irrelevant stimuli, which induced apparent motion of either the hand or the mouth on each trial.

The letters were in Arial font size 28, lowercase, and printed in white on a black background. The two letters combined occupied $1.0^{\circ}$ (width) $\times 2.4^{\circ}$ (height) of viewing angle. The hand and mouth stimuli were matched in terms of overall size of stimulus in the neutral position, size of stimulus when in the open position, and size of stimulus when in the closed position. In the neutral position, the hand occupied $3.3^{\circ}$ (width) $\times 5.7^{\circ}$ (height) of viewing angle, and the mouth occupied $3.3^{\circ}$ (width) $\times 5.2^{\circ}$ (height) of viewing angle. The distance between the middle finger and thumb when the hand was in the neutral position occupied $1.4^{\circ}$ of viewing angle, and the distance between the top and bottom lip when the mouth was in the neutral position occupied $1.6^{\circ}$ of viewing angle. The distance between the middle finger and thumb when the hand was in the open position occupied $5.2^{\circ}$ of viewing angle, and the distance between the top and bottom lip when the mouth was in the open position occupied $5.0^{\circ}$ of viewing angle. In the closed position, the distance between the lips and between the fingers occupied $0^{\circ}$ of viewing angle.

There were four letter pairs ( $h o, h c, m o$, and $m c$ ), each consisting of a letter indicating the correct response effector ( $h$ for hand or $m$ for mouth) and a letter indicating the correct response movement ( $o$ for open or $c$ for close). In half of the imperative stimuli the letter representing the effector was placed above the letter representing the movement, and in the other half, this configuration was reversed.

Data recording and analysis. For both open and closed responses, response onset was measured using disposable $\mathrm{Ag} / \mathrm{AgCl}$ surface electrodes by recording the electromyogram (EMG) from the first dorsal interosseus (FDI) muscle in the hand and the orbicularis oris $(\mathrm{OO})$ in the mouth. Recording electrodes were placed on the $\mathrm{OO}$ on the right-hand corner of the mouth and on the FDI on the right hand. Signals were amplified, high-pass filtered at $20 \mathrm{~Hz}$, mains-hum filtered at $50 \mathrm{~Hz}$, and digitized at $2.5 \mathrm{kHz}$. They were rectified and smoothed using a dual-pass Butterworth filter, with a cut-off frequency of $50 \mathrm{~Hz}$. Signals were not low-pass filtered. To define a baseline, we registered EMG activity for 100 ms when the participant was not moving at the beginning of each trial. A window of $20 \mathrm{~ms}$ was then shifted progressively over the raw data in 1-ms steps. Response onset was defined by the beginning of the first 20-ms window after the imperative stimulus in
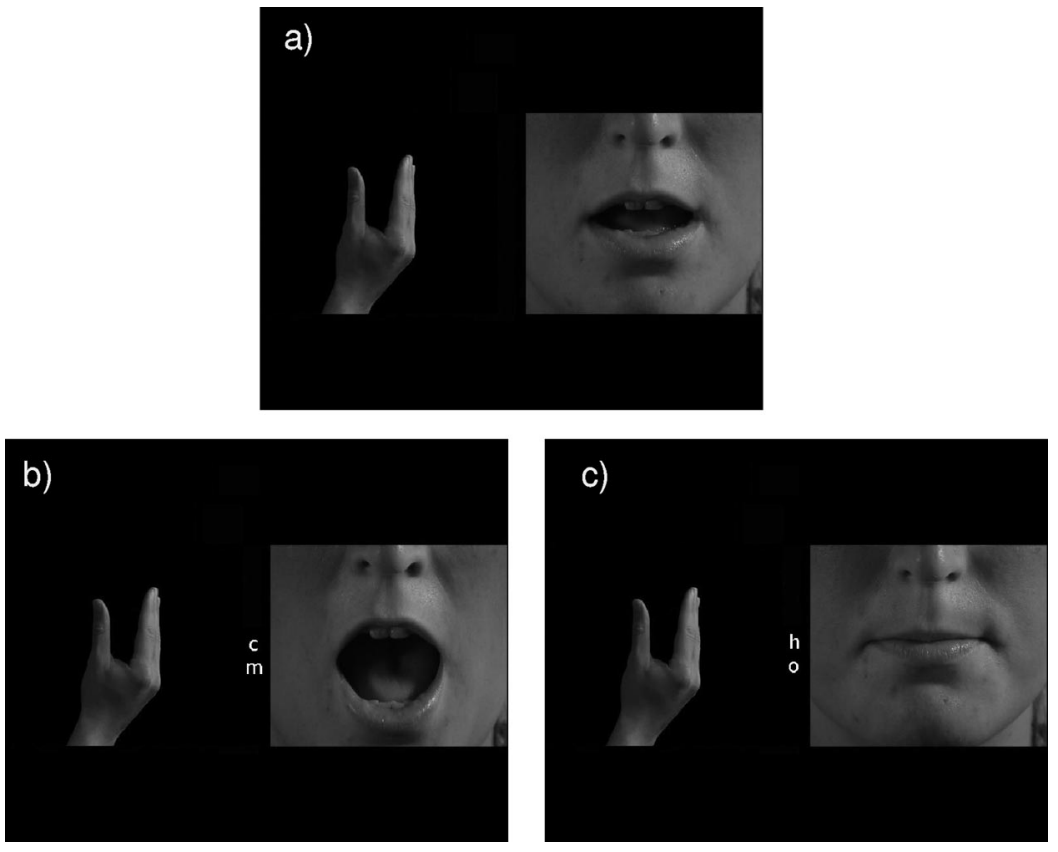

Figure 1. Stimuli depicting (a) the neutral warning stimulus and (b and c) two examples of imperative stimuli. In Figure $1 \mathrm{~b}$ the irrelevant stimulus (open mouth) is effector compatible and movement incompatible with the required response (closed mouth). In Figure 1c the irrelevant stimulus (closed mouth) is effector incompatible and movement incompatible with the required response (open hand). 
which the standard deviation for that window, and for the following 20-ms epoch, was greater than 2.75 times the standard deviation of the baseline. This criterion was chosen during initial calibration of the equipment as the most effective in discriminating false positives from misses. Whether the criterion correctly defined movement onset in the present experiment was verified by sight for every trial performed by each participant. Stimulus onset marked the beginning, and EMG onset marked the end, of the response time (RT) interval. Errors were recorded manually.

Procedure. Each participant was tested individually in a dimly lit room. Participants were told that they would see some pictures of hands and mouths on either side of the screen, but that they should ignore their movements and respond to the letters in the center of the screen. It was explained that they should open their mouth in response to the letters om, close their mouth in response to $\mathrm{cm}$, open their hand in response to $\mathrm{oh}$, and close their hand in response to $\mathrm{ch}$. Participants were told to make their movement as soon as the letter appeared on the screen but to keep as still as possible at all other times.

The participant's right forearm lay in a horizontal position across his or her body, parallel with the stimulus monitor. It was supported from elbow to wrist by an armrest such that the hand was free to move. The wrist was rotated so that the fingers moved upward during opening responses and downward when closing. Participants were shown the correct neutral positions for the hand and the mouth. In the neutral mouth position, participants were required to have their lips slightly parted. They were asked to return to the neutral position after they had made each movement.

All trials began with presentation of the warning stimulus, which was replaced $800-2000 \mathrm{~ms}$ later by an imperative stimulus of 480-ms duration. The stimulus onset asynchrony (SOA) varied randomly between 1200 and $2400 \mathrm{~ms}$ in 400-ms steps. After the imperative stimulus the screen went black for $3000 \mathrm{~ms}$ before the warning stimulus for the next trial appeared.

Each block contained 128 trials in total, 32 trials of each of the four principal types (effector and movement compatible; effector compatible and movement incompatible; effector incompatible and movement compatible; effector and movement incompatible) in random order. In half of the trials of each type, the hand stimulus was on the left of the mouth stimulus, and in the other half it was on the right. Each participant completed two blocks of trials, one in which the effector indicator ( $h$ or $m$ ) was above the movement indicator ( $o$ or $c$ ), and the other in which it was below the movement indicator. Half of the participants completed the blocks in the order described, and half in the alternative order. Before testing commenced in each block, participants completed 10 practice trials consisting of a random selection of trial types from within that block.

\section{Results and Discussion}

Practice trials, incorrect responses (3.9\%), and response omissions $(3.8 \%)$ were excluded from the analysis, as were all RTs smaller than $100 \mathrm{~ms}$ and greater than $1500 \mathrm{~ms}(0.05 \%)$. One participant, for whom more than $10 \%$ of the data were missing, was excluded from the analysis. The RT data from the remaining 12 participants are shown in the upper panel of Figure 2.

The data were subjected to analysis of variance (ANOVA) in which movement compatibility (compatible and incompatible),
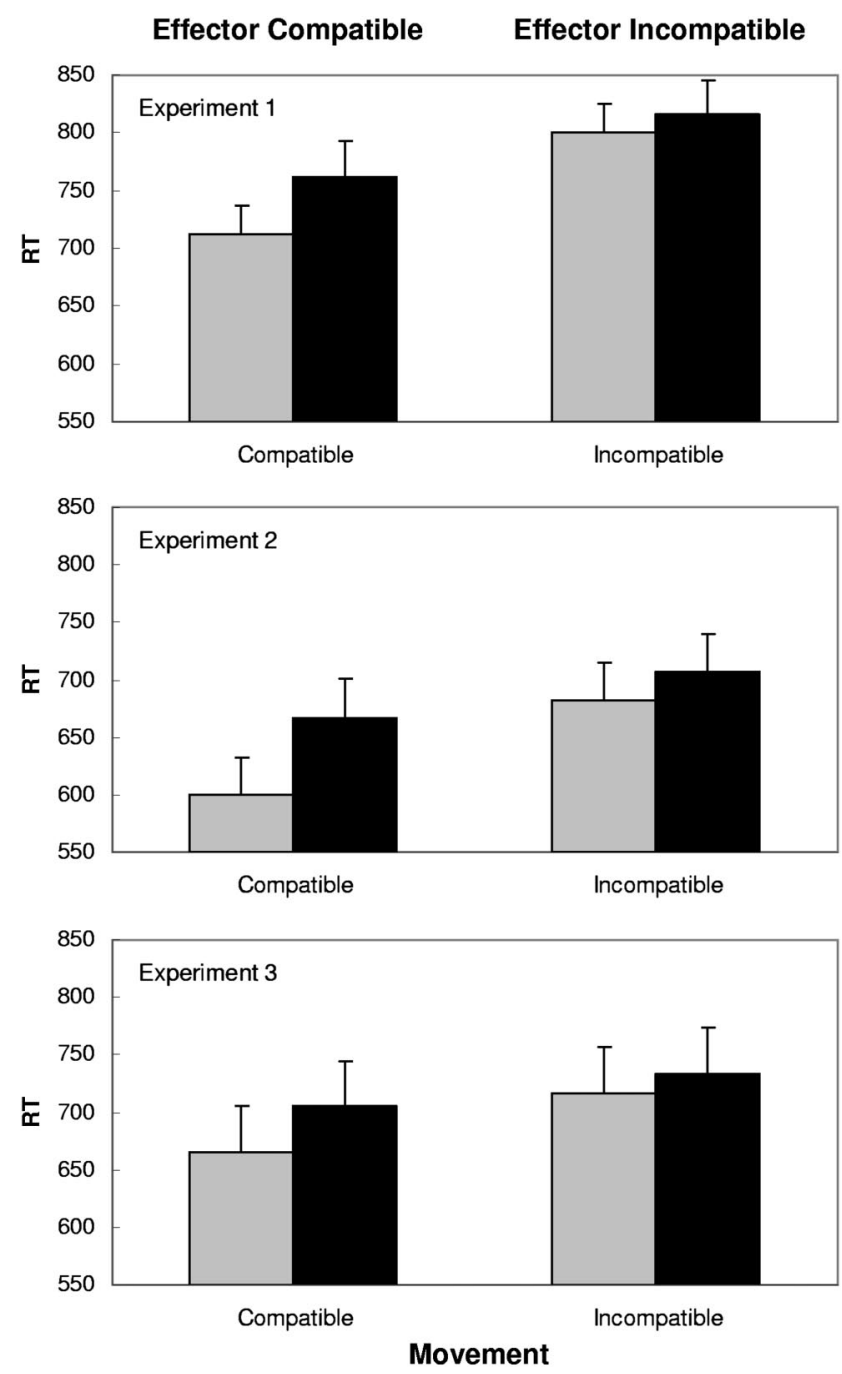

Figure 2. Mean reaction time (RT) on movement-compatible trials (black bars) and movement-incompatible trials (grey bars) when effector was compatible (left side) and when it was incompatible (right side), for Experiments 1 (upper panel), 2 (middle panel), and 3 (lower panel). Vertical bars indicate the standard error of the mean.

effector compatibility (compatible and incompatible), response movement (open and close), and response effector (mouth and hand) were within-subject variables. There were significant main effects of movement compatibility, $F(1,11)=45.1, p<.0001$, and effector compatibility, $F(1,11)=86.0, p<.0001$. Participants were faster to respond when the irrelevant stimulus movement was response compatible (mean: 752 SEM: 25) than when it was response incompatible $(M=788, S E M=27)$, and when the irrelevant stimulus effector was response compatible $(M=732$, SEM: 26) than when it was response incompatible $(M=809$, $S E M=27)$. There were also significant main effects of response effector, $F(1,11)=32.8, p<.0001$, and of response movement, $F(1,11)=21.7, p<.0001$. Shorter RTs were recorded for mouth responses $(M=728, S E M=27)$ than for hand responses $(M=$ $812, S E M=28)$. Shorter RTs were also recorded for open responses $(M=738, S E M=25)$ than for close responses $(M=802$, 
$S E M=29)$. Rather than reflecting genuine differences in response latencies, these main effects may be artifacts of electrode placement. The FDI muscle may become active toward the end of a closing movement but nearer the beginning of an opening movement. Similarly, OO muscle activation may occur earlier in the course of a mouth-opening movement than does FDI activation in the course of a hand-opening movement.

The effect of movement compatibility was greater for closing than for opening responses-Movement Compatibility $\times$ Response Movement, $F(1,11)=5.6, p<.04$-but simple effects analysis confirmed that movement-compatible responses were faster than were movement-incompatible responses both when the response was opening- $F(1,11)=11.2, p<.006$; compatible: $M=728, S E M=25$; incompatible: $M=749, S E M=25$ - and when it was closing $-F(1$, $11)=27.9, p<.0001$; compatible: $M=777, S E M=26$; incompatible: $M=827, S E M=32$. Similarly, the effect of effector compatibility was greater when responses were made with the hand than when they were made with the mouth-Effector Compatibility $\times$ Response Effector, $F(1,11)=6.8, p<.03$ - but effectorcompatible responses were faster both for mouth responses- $F(1$, $11)=42.4, p<.0001$; compatible: $M=698, S E M=26$; incompatible: $M=759, S E M=28$ - and for hand responses- $(F(1,11)=$ 69.5, $p<.0001$; compatible: $M=766, S E M=28$; incompatible: $M=859$, SEM $=28$.

Of principal interest, given the purpose of the experiment, there was a significant Movement Compatibility $\times$ Effector Compatibility interaction, $F(1,11)=5.2, p<.044$, indicating that the movement compatibility effect was greater in the effectorcompatible condition $(53 \mathrm{~ms})$ than in the effector-incompatible condition $(18 \mathrm{~ms})$. Simple effects analysis indicated a significant movement compatibility effect when the response effector was compatible with the stimulus effector, $F(1,11)=35.4, p<.0001$, but only a trend in this direction when the response effector was incompatible, $F(1,11)=3.5, p<.088$.

Thus, the results of the present experiment indicate that automatic imitation is not wholly effector independent. Opening and closing movements of an irrelevant action stimulus had more impact on the speed of opening and closing responses when the responses were performed with the modeled effector (hand responses to hand stimuli, and mouth responses to mouth stimuli) than when they were performed with the alternative effector (hand responses to mouth stimuli, and mouth responses to mouth stimuli). Therefore, they imply that the effector dependence of instructed imitation is not entirely conventional; it is likely to reflect the operation of core imitation mechanisms, and not to be due solely to inferences about what is expected or expedient in the test situation.

However, the results of Experiment 1 did not indicate clearly whether automatic imitation is partially or completely effector dependent. Partial effector dependence is suggested by the observation that movement-compatible responses with the incompatible effector were substantially faster than were movementincompatible responses with the incompatible effector, but this trend was not quite significant, $F(1,11)=3.51, p<.088$.

\section{Experiment 2}

The principal purpose of Experiment 2 was to establish more clearly whether automatic imitation is partially or completely effector dependent. Therefore, Experiment 2 replicated the basic design and procedure used in Experiment 1, but with an additional sample of participants. Experiment 2 had two further purposes: to check that the movement-compatibility effect observed in Experiment 1 was not dependent on spatial compatibility, and to investigate the nature of the effector compatibility effects observed in Experiment 1 .

In Experiment 1, hand stimuli moved in a horizontal plane (e.g., the fingers moved to the right of the screen when the hand opened), whereas hand and mouth responses were made in a vertical plane (e.g., the fingers and the upper lip moved upward when participants made hand-opening and mouth-opening responses, respectively). Therefore, in hand stimulus trials, the tendency to respond faster on movement-compatible trials (e.g., hand or mouth opening in response to a hand-open stimulus) could not have been due to spatial compatibility. However, in mouth stimulus trials, up-down spatial compatibility was confounded with movement compatibility. For example, in mouth-open stimulus trials, the upper lip of the mouth stimulus moved upward, and correct responses in the movement compatible condition involved upward movement of the participants' fingers or upper lip. To remove this confound, in Experiment 2 both the hand and the mouth stimuli moved in the horizontal plane. Thus, in relation to Experiment 1, the mouth stimulus was rotated $90^{\circ}$ anticlockwise from the canonical position, so that the upper lip appeared on the left of the lower lip, and moved toward the left when the mouth stimulus opened. As in Experiment 1, responses were made when the participant's head was upright. If the movement compatibility effect observed in Experiment 1 was not dependent on up-down spatial compatibility, then it should be replicated in Experiment 2, in which updown spatial compatibility was controlled.

The third purpose of Experiment 2 was to investigate the effector compatibility effects found in Experiment 1. These effects showed that responses with the modeled effector (e.g., hand responses to hand stimuli) were faster, and more susceptible to automatic imitation, than were responses with the alternative effector (e.g., hand responses to mouth stimuli). These effects might indicate that observation of an effector system in motion facilitates responding with an anatomically similar effector system, for example, hand movement observation facilitates hand movements in relation to mouth movements. However, these effects might indicate, instead or in addition, that observation of an effector system in motion facilitates responding at body-centered locations typically occupied by that effector system. For example, hand movement observation may facilitate responses made in the typical locations of the hands (e.g., close to the middle of the trunk when seated) in relation to responses made in the location of the mouth. To test the anatomical account against the response location account of effector compatibility, Experiment 2 varied the location of the response hand. In the far condition, as in Experiment 1, the participant's responding hand rested on the desk top, just in front of his or her solar plexus. In the near condition, the participant's responding hand was located directly in front of his or her mouth. If the effector compatibility effects observed in Experiment 1 were due to the locations of the responding effectors, and not to the anatomical relationship between the stimulus and response effectors, then those effects should be substantially smaller in the near condition than in the far condition.

It is also possible, in principle, that the effector compatibility observed in Experiment 1 was related to the fact that participants 
could see their hand responses, albeit in peripheral vision, whereas they could not see their mouth responses. To control for this, in Experiment 2 we placed a screen between the participant's body and his or her responding hand, so that neither hand nor mouth responses were accompanied by visual feedback.

\section{Method}

Participants. Twelve additional participants, with an average age of 28.2 years ( 7 men) were recruited from the same source, and to meet the same requirements, as in Experiment 1.

Stimuli. The stimuli were identical to those in Experiment 1 except that the mouth stimuli were rotated by $90^{\circ}$ in an anticlockwise direction from the canonical position, so that the upper lip appeared on the left of the lower lip, and moved toward the left when the mouth stimulus opened. As in Experiment 1, responses were made when the participant's head was upright. Therefore, for both hand and mouth stimuli, the direction of stimulus movement (left/right) was orthogonal to that of the response movement (up/ down).

Procedure. The data recording and analysis were identical to those in Experiment 1. The procedure was also the same as in Experiment 1, except as follows. Participants completed eight blocks of trials over two sessions, between 1 and 3 days apart. Half of the blocks were completed in the hand far position, where, as in Experiment 1, the hand was located approximately $400 \mathrm{~mm}$ below the mouth. The remaining blocks were completed in the hand near condition, where the participant's right arm lay in a horizontal position on a raised armrest such that the hand was as close as possible to the mouth in both the horizontal and vertical planes. In both conditions the hand was covered by a rigid black screen so that the participant could not see his or her movements. Therefore, in Experiment 2, neither hand nor mouth responses yielded visual feedback.

Each block contained 64 trials in total, 16 trials of each of the four principal types (effector and movement compatible; effector compatible and movement incompatible; effector incompatible and movement compatible; effector and movement incompatible) in random order. In half of the trials of each type, the hand stimulus was on the left of the mouth stimulus, and in the other half it was on the right. Each participant completed four blocks of trials on each day, two in which the hand was in the near position and two in which the hand was in the far position. In one near block the effector indicator ( $h$ or $m$ ) was above the movement indicator ( $o$ or $c$ ), and in the other near block it was below the movement indicator. This was also the case for the far blocks. The order of presentation of the blocks was counterbalanced. Participants carried out the same four blocks on both days, but on the second day the order of near and far blocks was reversed.

\section{Results and Discussion}

Practice trials, incorrect responses $(2.9 \%)$, and response omissions (3.0\%) were excluded from the analysis as were all RTs smaller than $100 \mathrm{~ms}$ and greater than $1500 \mathrm{~ms}(0.05 \%)$. The RT data for the 12 participants is shown in the middle panel of Figure 2. On average, RTs were faster in Experiment $2(M=$ $663.5, S E M=9.4)$ than in Experiment $1(M=778.8, S E M=8.5)$. Participants completed twice as many trials in Experiment 2 than in Experiment 1, and they were presented in shorter blocks. Therefore, the RT difference between experiments is likely to reflect the fact that participants in Experiment 2 had more practice and were less susceptible to fatigue.

The RT data were subjected to ANOVA in which movement compatibility (compatible and incompatible), effector compatibility (compatible and incompatible), response movement (open and closed), response effector (mouth and hand), and hand position (near and far) were within-subject variables. There were no significant main effects or interactions involving the hand position variable. There were significant main effects of movement compatibility, $F(1,11)=58.4 p<.0001$, and effector compatibility, $F(1,11)=39.0, p<.0001$. Participants were both faster to respond when the irrelevant stimulus movement was response compatible $(M=640, S E M=28)$ than when it was response incompatible $(M=687$, $S E M=32)$, and when the irrelevant stimulus effector was response compatible $(M=633, S E M=27)$ than when it was response incompatible $(M=694, S E M=33)$. There were also significant main effects of response movement, $F(1,11)=7.5, p<.02$, and of response effector, $F(1,11)=12.4$, $p<.005$. Shorter RTs were recorded for mouth responses $(M=$ $644, S E M=30)$ than for hand responses $(M=683, S E M=31)$, and for open responses $(M=650, S E M=28)$ than for close responses $(M=677, S E M=32)$.

The effect of movement compatibility was greater for closing responses than for opening responses (Movement Compatibility $\times$ Response Movement, $F(1,11)=11.4, p<.006$, but simple effects analysis confirmed that movement-compatible responses were faster than were movement-incompatible responses both when the response was opening $-F(1,11)=23.2, p<.001$; compatible: $M=633, S E M=28$; incompatible: $M=667, S E M=30$-and when it was closing $-F(1,11)=69.8, p<.0001$, compatible: $M=648, S E M=30$; incompatible: $M=706, S E M=34$. Similarly, the effect of effector compatibility was greater when responses were made with the hand than when they were made with the mouth (Effector Compatibility $\times$ Response Effector), $F(1,11)=5.2, p<.04$, but effector-compatible responses were faster both for mouth responses $-F(1,11)=29.5, p<.0001$; compatible: $M=620, S E M=28$; incompatible: $M=669, S E M=$ 33 - and for hand responses- $F(1,11)=32.8, p<.0001$; compatible: $M=647, S E M=27$; incompatible: $M=718, S E M=35$.

Of principal interest, there was a significant Movement Compatibility $\times$ Effector Compatibility interaction, $F(1,11)=13.4$, $p<.004$, indicating that the movement compatibility effect was greater in the effector-compatible condition $(68 \mathrm{~ms})$ than in the effector-incompatible condition $(25 \mathrm{~ms})$. Simple effects analysis indicated a significant movement compatibility effect when the response effector was compatible with the stimulus effector, $F(1$, $11)=64.7, p<.0001$, and also when the response effector was incompatible, $F(1,11)=8.7, p<.013$.

The results of the present experiment replicated and extended those of Experiment 1. As in Experiment 1, the results showed that movement compatibility has less influence on performance when the stimulus and response effectors are incompatible than when they are compatible. Additionally, by controlling for any effects of up-down spatial compatibility on movement compatibility, Experiment 2 confirmed that the hand and mouth movement compatibility effects observed in these experiments were genuine; they were due to the relationship between the stimulus and response 
actions (opening and closing), and not simply to the elementary spatial properties of these actions. Similarly, by controlling for the possibility that, for example, hand movement stimuli prime movements at canonical hand locations, rather than hand movements per se, Experiment 2 confirmed that the effector compatibility effects observed in these experiments were due to the anatomical, rather than to the spatial, relationship between the stimulus and response effectors. Most important, the results of Experiment 2 confirmed that, although the effect of movement compatibility is smaller when the stimulus and response effectors are incompatible than when they are compatible, the movement compatibility effect is significant in the effector-incompatible condition. Thus, automatic imitation of movement trajectory occurs even when participants are responding with hand movement to mouth movement stimuli, and vice versa. This finding suggests that the core mechanisms of imitation operate in a way that is partially, but not wholly, effector dependent.

\section{Experiment 3}

Experiment 3 examined the generality of the finding, reported in Experiments 1 and 2, that automatic imitation is partially effector dependent. It is possible that, in the previous experiments, we observed partial rather than complete effector dependence because the imperative stimuli consisted of the initial letters of the appropriate response (e.g., om indicated that the participant should make an open-mouth response), and thereby encouraged verbal coding, not only of the imperative stimulus, but also of the response and the task-irrelevant action stimulus. In this case, the transfer of the movement compatibility effect across effectors might have been due to the relations between elements of the verbal codes. For example, a task-irrelevant action stimulus coded as open hand might facilitate execution of a response coded as open mouth because the first element in each of these codes is the same. This example assumes that the movement compatibility effects observed in Experiments 1 and 2 were dependent on stimulusresponse (S-R) relations, that is, that verbal coding of the taskirrelevant stimulus had a direct effect on execution of the response. However, verbal coding of the imperative stimuli and taskirrelevant action stimuli would enhance their dimensional overlap (Kerzel \& Bekkering, 2000; Kornblum, 1992, 1994), and thereby raise the possibility that the movement compatibility effects observed in Experiments 1 and 2 were dependent on stimulusstimulus (S-S) rather than S-R relations (Hommel, 1993, 1995, 1997; Kornblum, Hasbroucq, \& Osman, 1990; Lu \& Proctor, 1995). For example, a task-irrelevant action stimulus coded as open hand might facilitate processing of an imperative stimulus coded open mouth because the first element in each of these stimulus codes is the same.

In Experiment 3, we used patches of color, rather than pairs of letters, as the imperative stimuli. If the results of Experiments 1 and 2 were dependent on verbal coding, encouraged by the use of verbal rather than arbitrary imperative stimuli, then one would not expect them to be replicated in Experiment 3. In contrast, if the results of Experiments 1 and 2 reflect S-R compatibility effects, and indicate that automatic imitation is partially effector dependent even when it is not verbally mediated, then one would expect the results of Experiment 3 to be essentially the same as those of the previous experiments.

\section{Method}

Participants. Twelve additional participants, with an average age of 28.7 years ( 5 men) were recruited from the same source, and to meet the same requirements, as in Experiments 1 and 2.

Stimuli. The stimuli were identical to those in Experiment 2 except that the imperative stimulus letters were replaced with a small colored rectangle. There were four colors: green, purple, blue, and yellow, which were in the same position and occupied approximately the same area as the letters in Experiments 1 and 2 $\left(1.0^{\circ}\right.$ wide $\times 2.4^{\circ}$ high of viewing angle).

Procedure. The data recording and analysis were identical to Experiments 1 and 2. The procedure was also the same as in Experiment 2, except as follows. Instead of letters, colors specified which response to make on each trial. Before the experiments commenced, participants were given a short period of training so they could learn the stimulus-response mappings. They were told that on each trial they would see one of four colored squares that would indicate which of the four movements they should make. They were given a list of the stimulus-response mappings and asked to memorize them. Following this, participants completed 40 practice trials in which they responded to a colored square in the center of the screen. On the practice trials, no irrelevant stimulus movement was present. In the test session, participants completed four blocks of 64 trials, with their response hand in the far position described in Experiment 2.

\section{Results and Discussion}

Practice trials, incorrect responses (3.1\%), and response omissions $(3.4 \%)$ were excluded from the analysis as were all RTs smaller than $100 \mathrm{~ms}$ and greater than $1500 \mathrm{~ms}(0.05 \%)$. The RT data for the 12 participants is shown in the lower panel of Figure 2. On average, RTs in Experiment $3(M=705, S E M=18)$ were slower than those in Experiment $2(M=664, S E M=9)$ and faster than those in Experiment $1(M=779, S E M=9)$. Participants completed twice as many trials in Experiment 2 than in Experiment 3. Therefore, the RT difference between these experiments is likely to reflect the fact that participants in Experiment 2 had more practice. Additionally, blocks were shorter in Experiment 3 than in Experiment 1, and therefore the RT difference between these experiments is likely to reflect the fact that participants in Experiment 3 were less susceptible to fatigue.

The RT data was subjected to ANOVA in which movement compatibility (compatible and incompatible), effector compatibility (compatible and incompatible), response movement (open and closed), and response effector (mouth and hand) were withinsubject variables. There were significant main effects of movement compatibility, $F(1,11)=9.9 p<.01$, and effector compatibility, $F(1,11)=8.4, p<.016$. Participants were faster to respond both when the irrelevant stimulus movement was response compatible $(M=685, S E M=30)$ than when it was response incompatible $(M=725, S E M=40)$, and when the irrelevant stimulus effector was response compatible $(M=690, S E M=36)$ than when it was response incompatible $(M=720, S E M=34)$. There was a significant main effect of response movement, $F(1,11)=6.5, p<$ .029. Shorter RTs were recorded for open responses $(M=668$, $S E M=32)$ than for closed responses $(M=702, S E M=33)$.

Of principal interest, there was a significant Movement Compatibility $\times$ Effector Compatibility interaction, $F(1,11)=8.9$, 
$p<.012$, indicating that the movement compatibility effect was greater in the effector-compatible condition $(41 \mathrm{~ms})$ than in the effector-incompatible condition $(17 \mathrm{~ms})$. Simple effects analysis indicated a significant movement compatibility effect when the response effector was compatible with the stimulus effector, $F(1$, $11)=4.61, p<.001$, and also when the response effector was incompatible, $F(1,11)=2.7, p<.022$.

The use of color patches rather than initial letters as the imperative stimuli in Experiment 3 substantially reduced the risk that participants would engage in verbal coding of those stimuli and of other elements of the task. Therefore, the fact that partial effector dependence was observed in this experiment, as it was in Experiments 1 and 2, suggests that this phenomenon does not rely on verbal mediation. Instead, it appears to be a general property of automatic imitation.

\section{General Discussion}

In everyday life, and in laboratory tasks in which participants are instructed to imitate, movements are usually imitated with the modeled effector system. To investigate whether this effectordependent tendency in imitative performance reflects the nature of the core mechanisms of imitation, we used a SRC paradigm to investigate the effector dependence of automatic imitation. The results of three experiments indicated an automatic imitation, or movement compatibility, effect both when participants responded with the modeled effector system (effector-compatible condition) and when they responded with an alternative effector system (effector-incompatible condition), and that the movement compatibility effect was smaller when an alternative effector was used. More specifically, opening and closing movements of an irrelevant action stimulus had more impact on the speed of opening and closing responses when the responses were performed with the modeled effector (hand responses to hand stimuli, and mouth responses to mouth stimuli) than when they were performed with the alternative effector (hand responses to mouth stimuli, and mouth responses to mouth stimuli).

Previous studies have demonstrated movement compatibility effects for hand opening and closing stimuli when the stimulus and response effectors were compatible (Heyes et al., 2005; Press et al., 2005; Stürmer et al., 2000), but this study shows for the first time that automatic imitation of opening and closing movements of the hand and mouth transfers across these effector systems, and that the transfer is incomplete; automatic imitation of opening and closing movements of the hand and mouth is partially effector dependent.

As its name suggests, automatic imitation is likely to be less susceptible to cognitive control than to imitative performance on the basis of instructions or endogenously generated intentions (Press, Gillmeister, \& Heyes, 2006). Therefore, the effector dependence of automatic imitation observed in the present study suggests that, in instructed imitation tasks and in everyday life, participants do not imitate with the modeled effector purely because they judge effector matching to be conventional or expedient. Rather, it suggests that the effector dependence of imitative performance reflects the structure of the core neurocognitive mechanisms of imitation, the mechanisms that solve the "correspondence problem" (Brass \& Heyes, 2005) by translating visual input from observed body movements into matching motor output.
Partial effector dependence of automatic imitation is consistent with the associative sequence learning (ASL; Heyes, 2001; Heyes \& Ray, 2000) model of imitation, which suggests that visual input from the model is translated into motor output by a set of bidirectional, excitatory links connecting visual and motor representations of the same action components. These "vertical associations" are thought to be established through associative learning, formed on the basis of correlated experience of observing and executing action components. Because stimulus generalization is a ubiquitous feature of associative learning (Pearce, 1987, 1994), the ASL model assumes that vertical associations are activated, not only by the stimuli experienced during learning, but also by other stimuli to the extent that they have physical characteristics in common with the learned stimulus (Press et al., 2005, 2006). Therefore, the ASL hypothesis predicts some facilitation of mouth opening by observation of hand opening, and vice versa, given the visual similarity between the opening movement of a mouth and the opening movement of a hand.

Partial effector dependence of imitation is also consistent with ideomotor theory, the theory of event coding, and the concept of dimensional overlap (Greenwald, 1970; Hommel, Musseler, Aschersleben, \& Prinz, 2001; Kornblum et al., 1990; Prinz, 1997, 2002). These theories suggest that actions are represented in terms of their sensory consequences, and that action observation primes performance of the same action to the extent that the observed and executed actions have similar sensory consequences. This view can readily explain automatic imitation of hand movements because they are perceptually transparent (Heyes \& Ray, 2000), that is, they yield similar visual effects when observed and executed. Automatic imitation of mouth movements is harder to reconcile with ideomotor theory. This is because mouth movements are perceptually opaque; they yield dissimilar sensory input when observed and executed. For example, when I open my mouth, I do not receive any distinctive visual input, but when I observe someone else opening his or her mouth, I see the lips parting and forming an oval shape. However, ideomotor theory is able to explain imitation of perceptually opaque actions, and partial effector dependence of automatic imitation, when it is combined with the hypothesis, central to the ASL model, that actions acquire their "common," sensory codes through associative learning (Brass \& Heyes, 2005; Elsner \& Hommel, 2001, 2004).

Partial, rather than complete, effector dependence is apparently inconsistent with the active intermodal matching (AIM) theory of imitation ( Meltzoff \& Moore, 1997). This theory suggests that the mechanisms that solve the correspondence problem operate in two stages: "an imitative act is not one indissociable unit. It can be differentiated into organ [effector] identification and movement components" (Meltzoff \& Moore, 1997, p. 184). The first stage identifies the modeled effector, and when it is completed, the second stage codes the movement performed by that effector. This account implies that the mechanism mediating movement imitation, operative in the second stage, is strictly effector dependentthat it represents the modeled movement in codes that apply exclusively to the modeled effector-and therefore that automatic imitation should be wholly, rather than partially, effector dependent.

It is likely that, at the neurological level, imitation is mediated by cortical areas with "mirror" properties, areas that are active both when an action is passively observed and when the same action is 
executed without visual feedback (e.g., Iacoboni et al., 1999). "Mirror neurons," single units with these visuomotor properties, were first discovered in the premotor and parietal cortices of macaques (e.g., Gallese, Fadiga, Fogassi, \& Rizzolati, 1996; Keysers et al., 2003; Rizzolatti, Fadiga, Gallese, \& Fogassi, 1996), and there is now a substantial body of evidence suggesting that there are analogous or homologous areas in the human brain. These studies have revealed mirror areas of premotor and parietal cortices that are sensitive to movement type-active when a movement with a particular topography or trajectory is observer and executed (Gallese et al., 1996; Rizzolatti et al., 1996) — and to effector type-active when a movement involving a particular effector is observed and executed (Aziz-Zadeh, Maeda, Zaidel, Mazziotta, \& Iacoboni, 2002; Buccino et al., 2001; Fadiga, Fogassi, Pavesi, \& Rizzolatti, 1995; Strafella \& Paus, 2000; Watkins, Strafella, \& Paus, 2003; Wheaton, Pipingas, Silberstein, \& Puce, 2001; Wheaton, Thompson, Syngeniotis, Abbot, \& Puce, 2004).

Recent research indicating that the development of mirror areas depends on learning (Calvo-Merino, Glaser, Grezes, Passingham, \& Haggard, 2005; Cross, Hamilton, \& Grafton, 2006; Ferrari, Rozzi, \& Fogassi, 2005; Keysers et al., 2003), and especially on sensorimotor learning (Catmur, Gillmeister, Bird, Liepelt, Brass, \& Heyes, 2008; Catmur, Walsh, \& Heyes, 2007), has promoted the integration of neurological and cognitive models of imitation. More specifically, it has raised the possibility that mirror neurons or areas are formed through correlated experience of observing and executing actions, and therefore that they are the neural correlates of the vertical associations postulated by the ASL model (Heyes, 2005; Heyes, 2010).

In conclusion, the results of the present study indicate that automatic imitation is partially effector dependent: Observation of opening and closing movements of the hand and mouth prime execution of corresponding movements by the modeled effector and also by the alternative effector, but the priming effect is smaller when the alternative effector is engaged in responding. This finding, which is consistent with the ASL and ideomotor theories of imitation, suggests that rather than being conventional, the effector dependence of intentional and instructed imitation reflects the nature of the mechanisms that mediate visuomotor translation for imitation.

\section{References}

Anisfeld, M. (1991). Neonatal imitation: Review. Developmental Review, $11,60-97$

Anisfeld, M. (1996). Only tongue protrusion modeling is matched by neonates. Developmental Review, 16, 149-161.

Aziz-Zadeh, L., Maeda, F., Zaidel, E., Mazziotta, J., \& Iacoboni, M. (2002). Lateralisation in motor facilitation during action observation: A TMS study. Experimental Brain Research, 144, 127-131.

Bach, P., \& Tipper, S. P. (2007). Implicit action encoding influences personal-trait judgments. Cognition, 102, 151-178.

Berger, S. M., \& Hadley, S. W. (1975). Some effects of a model's performance on an observer's electromyographic activity. American Journal of Psychology, 88, 263-276.

Bertenthal, B. I., Longo, M. R., \& Kosobud, A. (2006). Imitative response tendencies following observation of intransitive actions. Journal of Experimental Psychology: Human Perception and Performance, 32, $210-225$.

Bird, G., Brindley, R., Leighton, J., \& Heyes, C. M. (2007). General processes, rather than 'goals,' explain imitation errors. Journal of Experimental Psychology: Human Perception and Performance, 33, 1158 1169.

Brass, M., Bekkering, H., Wohlschlager, A., \& Prinz, W. (2000). Compatibility between observed and executed finger movements: Comparing symbolic, spatial, and imitative cues. Brain and Cognition, 44, 124-143.

Brass, M., \& Heyes, C. M. (2005). Imitation: Is cognitive neuroscience solving the correspondence problem? Trends in Cognitive Sciences, 9, 489-495.

Buccino, G., Binkofski, F., Fink, G. R., Fadiga, L., Fogassi, L., Gallese, V., ... Freund, H. J. (2001). Action observation activates premotor and parietal areas in a somatotopic manner: An fMRI study. European Journal of Neuroscience, 13, 400-404.

Calvo-Merino, B., Glaser, D. E., Grezes, J., Passingham, R. E., \& Haggard, P. (2005). Action observation and acquired motor skills: An fMRI study with expert dancers. Cerebral Cortex, 15, 1243-1249.

Catmur, C., Gillmeister, H., Bird, G., Liepelt, R., Brass, M., \& Heyes, C. (2008). Through the looking glass: Counter-mirror activation following incompatible sensorimotor learning. European Journal of Neuroscience, 28(6), 1208-1215.

Catmur, C., Walsh, V., \& Heyes, C. M. (2007). Sensorimotor learning configures the human mirror system. Current Biology, 17, 1527-1531.

Chartrand, T. L., \& Bargh, J. A. (1999). The chameleon effect: The perception-behaviour link and social interaction. Journal of Personality and Social Psychology, 76, 893-910.

Craighero, L., Fadiga, L., Rizzolatti, G., \& Umilta, C. (1998). Visuomotor priming. Visual Cognition, 5, 109-125.

Cross, E. S., Hamilton, A. F. C., \& Grafton, S. T. (2006). Building a motor simulation de novo: Observation of dance by dancers. NeuroImage, 31, $1257-1267$.

Elsner, B., \& Hommel, B. (2001). Effect anticipation and action control. Journal of Experimental Psychology: Human Perception and Performance, 27, 229-240.

Elsner, B., \& Hommel, B. (2004). Contiguity and contingency in actioneffect learning. Psychological Research, 68, 138-154.

Fadiga, L., Fogassi, L., Pavesi, G., \& Rizzolatti, G. (1995). Motor facilitation during action observation: A magnetic stimulation study. Journal of Neurophysiology, 73, 2608-2611.

Ferrari, P. F., Rozzi, S., \& Fogassi, L. (2005). Mirror neurons responding to observation of actions made with tools in monkey ventral premotor cortex. Journal of Cognitive Neuroscience, 17, 212-226.

Franz, E. A., Ford, S., \& Werner, S. (2007). Brain and cognitive processes of imitation in bimanual situations: Making inferences about mirror neuron systems. Brain Research, 11, 138-149.

Gallese, V., Fadiga, L., Fogassi, L., \& Rizzolatti, G. (1996). Action recognition in the premotor cortex. Brain, 119, 593-609.

Gillmeister, H., Catmur, C., Brass, M., \& Heyes, C. M. (2010). Experiencebased priming of body parts: A study of imitation and the mirror system. Unpublished manuscript.

Greenwald, A. G. (1970). Sensory feedback mechanisms in performance control: With special reference to the ideo-motor mechanism. Psychological Review, 77, 73-99.

Head, H. (1920). Aphasia and kindred disorders of speech. Brain, 43, $87-165$.

Heyes, C. (2001). Causes and consequences of imitation. Trends in Cognitive Sciences, 5, 253-261.

Heyes, C. M. (2010). Where do mirror neurons come from? Neuroscience and Biobehavioral Reviews, 34, 575-583.

Heyes, C. M. (2005). Imitation by association. In S. Hurley \& N. Chater (Eds.), Perspectives on imitation: From Neuroscience to social science (pp. 157-176). Cambridge, MA: MIT Press.

Heyes, C. M., Bird, G., Johnson, H., \& Haggard, P. (2005). Experience modulates automatic imitation. Cognitive Brain Research, 22, 233-240. 
Heyes, C. M., \& Ray, E. (2000). What is the significance of imitation in animals? Advances in the Study of Behavior, 29, 215-245.

Heyes, C. M., \& Ray, E. D. (2004). Spatial S-R compatibility effects in an intentional imitation task. Psychonomic Bulletin \& Review, 11, 703-705.

Hommel, B. (1993). The relationship between stimulus processing and response selection in the Simon task: Evidence for a temporal overlap. Psychological Research, 55, 280-290.

Hommel, B. (1995). Stimulus-response compatibility and the Simon effect: Toward an empirical clarification. Journal of Experimental Psychology: Human Perception and Performance, 21, 764-775.

Hommel, B. (1997). Toward an action-concept model of stimulus-response compatibility. In B. Hommel \& W. Prinz (Eds.), Theoretical issues in stimulus-response compatibility (pp. 281-330). Amsterdam, The Netherlands: Elsevier.

Hommel, B., Musseler, J., Aschersleben, G., \& Prinz, W. (2001). The theory of event coding (TEC): A framework for perception and action planning. Behavioral and Brain Sciences, 24, 849-878.

Iacoboni, M., Woods, R. P., Brass, M., Bekkering, H., Mazziota, J. C., \& Rizzolatti, G. (1999). Cortical mechanisms of human imitation. Science, 286, 2526-2528.

Jones, S. S. (1996). Imitation or exploration? Young infants' matching of adults' oral gestures. Child Development, 67, 1952-1969.

Jones, S. S. (2006). Newborn imitation or arousal? The effect of music on four-week-old infants' tongue protrusions. Infant Behavior and Development, 29, 126-130.

Kerzel, D., \& Bekkering, H. (2000). Motor activation from visible speech: Evidence from stimulus-response compatibility. Journal of Experimental Psychology: Human Perception and Performance, 26, 634-647.

Keysers, C., Kohler, E., Umilta, M. A., Nanetti, L., Fogassi, L., \& Gallese, V. (2003). Audiovisual mirror neurons and action recognition. Experimental Brain Research, 153, 628-636.

Keysers, C., \& Perrett, D. I. (2004). Demystifying social cognition: A Hebbian perspective. Trends in Cognitive Sciences, 8, 501-507.

Kilner, J. M., Paulignan, Y., \& Blakemore, S. J. (2003). An interference effect of observed biological movement on action. Current Biology, 13, 522-525.

Kornblum, S. (1992). Dimensional overlap and dimensional relevance in stimulus-response and stimulus-stimulus compatibility. In G. E. Stelmach \& J. Requin (Eds.), Tutorials in motor behavior II (pp. 743-777). Amsterdam, The Netherlands: Elsevier

Kornblum, S. (1994). The way irrelevant dimensions are processed depends on what they overlap with: The case of Stroop and Simon-like stimuli. Psychological Research, 56, 130-135.

Kornblum, S., Hasbroucq, T., \& Osman, A. (1990). Dimensional overlap: Cognitive basis for stimulus response compatibility-A model and taxonomy. Psychological Review, 97, 253-270.

Lakin, J. L., \& Chartrand, T. L. (2003). Using nonconscious behavioral mimicry to create affiliation and rapport. Psychological Science, 14(4), 334-339.

Lu, C.-H., \& Proctor, R. W. (1995). The influence of irrelevant location information on performance: A review of the Simon and spatial Stroop effects. Psychonomic Bulletin and Review, 2, 174-207.

Meltzoff, A. N., \& Moore, M. K. (1977). Imitation of facial and manual gestures by human neonates. Science, 198, 75-78.

Meltzoff, A. N., \& Moore, M. K. (1983). Newborn infants imitate adult facial gestures. Child Development, 54, 702-709.

Meltzoff, A. N., \& Moore, M. K. (1989). Imitation in newborn infants: Exploring the range of gestures imitated and the underlying mechanisms. Developmental Psychology, 25, 954-962.
Meltzoff, A. N., \& Moore, M. K. (1997). Explaining facial imitation: A theoretical model. Early Development and Parenting, 6, 179-192.

Pearce, J. M. (1987). A model of stimulus generalization for Pavlovian conditioning. Psychological Review, 94, 61-73.

Pearce, J. M. (1994). Similarity and discrimination: A selective review and a connectionist model. Psychological Review, 101, 587-607.

Press, C., Bird, G., Flach, R., \& Heyes, C. M. (2005). Robotic movement elicits automatic imitation. Cognitive Brain Research, 25, 632-640.

Press, C., Bird, G., Walsh, E., \& Heyes, C. M. (2008). Are intransitive actions automatically imitated? Brain \& Cognition, 67, 44-50.

Press, C., Gillmeister, H., \& Heyes, C. M. (2006). Bottom-up, not topdown, modulation of imitation by human and robotic models. European Journal of Neuroscience, 24, 2415-2419.

Prinz, W. (1997). Perception and action planning. European Journal of Cognitive Psychology, 9, 129-154.

Prinz, W. (2002). Experimental approaches to imitation. In A. N. Meltzoff \& W. Prinz (Eds.), The imitative mind: Development, evolution, and brain bases (pp. 143-163). Cambridge, England: Cambridge University Press.

Rizzolatti, G., Fadiga, L., Gallese, V., \& Fogassi, L. (1996). Premotor cortex and the recognition of motor actions. Cognitive Brain Research, $3,131-141$

Stanley, J., Gowen, E., \& Miall, R. C. (in press). Effects of agency on movement interference during observation of a moving dot stimulus. Journal of Experimental Psychology: Human Perception and Performance.

Stürmer, B., Aschersleben, G., \& Prinz, W. (2000). Effects of correspondence between complex stimulus and response patterns. Journal of Experimental Psychology: Human Perception and Performance, 26, $1746-1759$.

Strafella, A. P., \& Paus, T. (2000). Modulation of cortical excitability during action observation: A transcranial magnetic stimulation study. Neuroreport, 11, 2289-2292.

Tomasello, M. (1996). Do apes ape? In C. M. Heyes \& B. G. Galef, Jr. (Eds.), Social learning in animals: The roots of culture (pp. 319-346). San Diego, CA: Academic Press.

Ullstadius, E. (1998). Neonatal imitation in a mother-infant setting. Early Development and Parenting, 7, 1-8.

Wapner, S., \& Cirillo, L. (1968). Imitation of a model's hand movements: Age changes in transposition of left-right relations. Child Development, 39, 887-894.

Watkins, K. E., Strafella, A. P., \& Paus, T. (2003). Seeing and hearing speech excites the motor system involved in speech production. Neuropsychologia, 41, 989-994.

Wheaton, K., Pipingas, A., Silberstein, R., \& Puce, A. (2001). Human neural responses elicited to observing the actions of others. Visual Neuroscience, 18, 401-406.

Wheaton, K. J., Thompson, J. C., Syngeniotis, A., Abbott, D. F., \& Puce, A. (2004). Viewing the motion of human body parts activates different regions of premotor, temporal, and parietal cortex. Neurolmage, 22, 277-288.

Wohlschläger, A., Gattis, M., \& Bekkering, H. (2003). Action generation and action perception in imitation: An instance of the ideomotor principle. Philosophical Transactions of the Royal Society of London B, 358(1431), 501-515.

Received July 6, 2007

Revision received October 22, 2008 Accepted November 4, 2008 